\title{
Comparison of polypharmacy using low-dose second-generation antipsychotics plus low-dose first-generation antipsychotics with monotherapy using therapeutic-dose second-generation antipsychotics in schizophrenia - a pooled analysis
}

\author{
Ching-Hua Lin, ${ }^{1,2 *}$ (C) Fu-Chiang Wang, ${ }^{1}$ Yu-Hui Huang, ${ }^{1}$ Shih-Chi Lin, ${ }^{\prime}$ and \\ Chao-Chan Kuo'
}

\footnotetext{
${ }^{1}$ Kaohsiung Municipal Kai-Syuan Psychiatric Hospital, Kaohsiung, Taiwan

${ }^{2}$ Department of Psychiatry, School of Medicine, College of Medicine, Kaohsiung Medical University, Kaohsiung, Taiwan
}

Received 24 February 2019; Accepted 4 March 2019; First published 14 May 2019

Key words: first generation antipsychotics, second generation antipsychotics, antipsychotic polypharmacy, antipsychotic monotherapy, schizophrenia.

To the editor:

Antipsychotics were classified according to the risk of extrapyramidal side effects (EPS) as first-generation antipsychotics (FGA) (e.g. haloperidol, sulpiride, and trifluoperazine), or second-generation antipsychotics (SGA) (e.g. olanzapine, risperidone, and amisulpride) if the risk for the development of EPS is low. Treatment guidelines for schizophrenia favor antipsychotic monotherapy. However, antipsychotic polypharmacy is a common clinical practice. The rate of polypharmacy has increased over the past decade. ${ }^{1}$ To date, evidence supporting this practice is still mixed or inconclusive. Polypharmacy may maintain efficacy by additively blocking D2 receptors. An FGA at dosages lower than $600 \mathrm{mg}$ /day of chlorpromazine equivalents may have no more EPS than SGAs. ${ }^{2}$

We conducted a pooled analysis from three published 6-week, fixed dose, randomized, double-blind trials to explore whether polypharmacy using low-dose SGAs plus low-dose FGAs had efficacy and safety comparable with monotherapy using therapeutic-dose SGA in the acute treatment of 270 schizophrenia patients. For Trial 1 (NCT00998608), patients were randomly assigned to a $1: 1$ ratio of $2 \mathrm{mg} /$ day of risperidone plus $2 \mathrm{mg} /$ day of haloperidol or $4 \mathrm{mg} /$ day of risperidone alone for 6 weeks.

*Address correspondence to: Ching-Hua Lin, Department of Adult Psychiatry, Kaohsiung Municipal Kai-Syuan Psychiatric Hospital, 130, Kai-Syuan 2nd Rd., Ling-Ya District, Kaohsiung 802, Taiwan. (Email: chua.lin@msa.hinet.net)
For Trial 2 (NCT01615185), patients were randomly assigned to a 1:1 ratio of $400 \mathrm{mg} /$ day of amisulpride plus $800 \mathrm{mg} /$ day of sulpiride or $800 \mathrm{mg} /$ day of amisulpride alone for 6 weeks. For Trial 3 (NCT02704962), patients were randomly assigned to a $1: 1$ ratio of $5 \mathrm{mg} /$ day of olanzapine plus $5 \mathrm{mg}$ /day of trifluoperazine or $10 \mathrm{mg}$ /day of olanzapine alone for 6 weeks. Benzodiazepine p.r.n. and biperiden, up to $6 \mathrm{mg} /$ day, were also allowed for clinical requirement. Three trials were similar in design and were therefore pooled together. Efficacy measures, including the Positive and Negative Syndrome Scale (PANSS) and other scales, safety measures, side effect measures, and quality of life, were rated at baseline, and again at weeks $1,2,3,4$, and 6 (or upon early termination) or at baseline and again at endpoint. The response was defined as at least a 30\% reduction in the PANSS total score. Remission was defined according to "Andreasen criteria."

Patients were included in the analyses only if they had both a baseline and at least one post-baseline rating. Missing data were imputed according to the principle of last observation carried forward. Analyses of group differences were performed by analysis of covariance, with treatment as a fixed factor and sex, age, age at onset, and baseline values as covariates. Kaplan--Meier analysis was used to determine time to discontinuation, time to response, and time to remission between the two groups. Significance was defined as an alpha $<0.05$.

Polypharmacy $(n=138)$ and monotherapy groups $(n=132)$ were similar in (1) sex (male: $55.8 \%[n=77)]$ 
vs. $51.5 \%[n=68]$; female: $44.2 \%[n=61]$ vs. $48.5 \%$ $[n=64], p=0.48)$, age $(38.1 \pm 8.7$ vs. $39.0 \pm 8.2$, $p=0.85)$, age at onset $(24.6 \pm 6.9$ vs. $25.4 \pm 8.3, p=0.37)$, baseline scores of rating instruments, and baseline laboratory measures; (2) score changes in all efficacy measures, safety measures, side effect measures and quality of life (Supplementary Tables S1-S4), and (3) discontinuation rates $(26.8 \%$ [ $n=37]$ vs. $28.8 \%$ [ $n=38]$, $p=0.72)$, response rates $(35.5 \%[n=49]$ vs. $33.3 \%$ $[n=44], p=0.71)$, and remission rates $(40.6 \%[n=56]$ vs. $40.9 \%[n=54], \mathrm{p}=0.96)$. There were no significant differences in time to discontinuation (mean survival time $=34.5$ days vs. 34.8 days; $\log \operatorname{rank}=0.11, \mathrm{df}=1$, $p=0.74$ ), time to response (mean survival time $=37.4$ days vs. 37.3 days; $\log$ rank $=0.123, \mathrm{df}=1, p=0.73$ ), time to remission (mean survival time $=32.6$ days vs. 31.2 days; $\log$ rank $=0.321, \quad \mathrm{df}=1, \quad p=0.57)$, percentage of biperiden use $(22.5 \% \quad[n=31]$ vs. $23.5 \% \quad[n=31]$, $p=0.84$ ), and percentage of benzodiazepines use (86.2\% [ $n=119]$ vs. $82.6 \%[n=109], p=0.407)$ between the polypharmacy and monotherapy groups.

This study found that polypharmacy group had a degree of efficacy and safety comparable to monotherapy group. Compared to monotherapy, polypharmacy has been criticized for being associated with excessive dosing that may increase the risk of adverse events and increase treatment costs. ${ }^{3}$ The current study suggests that antipsychotic polypharmacy, with appropriate combinations, is as efficacious and safe as therapeutic-dose antipsychotic monotherapy in the treatment of schizophrenia. Brain image studies have revealed the presence of a therapeutic window of $60 \%$ to $80 \%$ dopamine D2 receptor occupancy of antipsychotics, which is associated with clinical effect without significantly increasing the risk of EPS. ${ }^{4}$ If polypharmacy, the combination of an appropriate ratio of one FGA dosage to another SGA dosage, were to reach $60 \%-80 \%$ D2 receptor occupancy, responses and EPS comparable to monotherapy could be achieved. However, the level of D2 occupancy of antipsychotic polypharmacy should be confirmed by imaging study. FGAs are much less expensive than SGAs. For patients in low- or middle-income countries or those without insurance coverage, the cost of drugs is a major barrier to treatment access. ${ }^{5}$ The finding of this study may provide evidence for a means of improving access to antipsychotics. Further longer-term, blinded studies with multiple outcomes measures are, however, needed.

\section{Disclosures}

The authors declare they have nothing to disclose.

\section{Supplementary material}

To view supplementary material for this article, please visit https://doi.org/10.1017/S1092852919000877

\section{REFERENCES:}

1. Ritsner MS. Polypharmacy in Psychiatry Practice. New York: Springer; 2013.

2. Leucht S, Wahlbeck $\mathrm{K}$, Hamann $\mathrm{J}$, et al. New generation antipsychotics versus low-potency conventional antipsychotics: a systematic review and meta-analysis. Lancet. 2003; 361(9369): 1581-1589.

3. Galling B, Roldán A, Rietschel L, et al. Safety and tolerability of antipsychotic co-treatment in patients with schizophrenia: results from a systematic review and meta-analysis of randomized controlled trials. Expert Opin Drug Saf. 2016; 15(5): 591-612.

4. Stahl SM. Stahl's Essential Psychopharmacology: Neuroscientific Basis and Practical Application. 4th ed. Cambridge, UK: Cambridge University Press; 2013.

5. Padmanathan P, Rai D. Access and rational use of psychotropic medications in low- and middle-income countries. Epidemiol Psychiatr Sci. 2016;25(1):4-8. 